\title{
Prevalence and Determinants of Dating Violence: An Umbrella Review of
}

\section{Systematic Reviews and Meta-analyses}

\author{
Md Mahbub Hossain*, ${ }^{1}$ Abida Sultana, ${ }^{2}$ Qiping Fan, ${ }^{1}$ Ping Ma, ${ }^{1}$ Neetu Purohit ${ }^{3}$
}

\begin{abstract}
Objective: Dating violence (DV) is a psychosocial problem which impacts the health and wellbeing of affected individuals. Many studies have reported a high prevalence and various determinants of DV; however, there is a gap of consolidated evidence on this problem. This umbrella review aimed to synthesize the current evidence on the prevalence and determinants of DV from systematic reviews and meta-analyses.

Methods: We systematically searched ten major databases and additional sources to retrieve systematic reviews or meta-analyses reporting prevalence or determinants of DV, published as peer-reviewed journal articles in English language till October 13, 2019. We extracted and synthesized the findings, reported the prevalence of DV, and categorized the determinants of DV using the socioecological model.

Results: We found 16 eligible systematic reviews and meta-analyses. Most of these studies were conducted in developed countries with a majority of adolescent and young adult participants. Studies reported varying prevalence of physical, sexual, psychological/emotional, and technology-assisted DV ranging from $0.1 \%$ to $57.5 \%, 0.1 \%$ to $64.6 \%, 4.2 \%$ to $97 \%$, and $5.8 \%$ to $92 \%$, respectively. The determinants of DV at different socioecological levels, including individual behavior, substance abuse, psychiatric conditions, experiencing violence and maltreatment; interpersonal factors like family and peer relationships; community and neighborhood characteristics; patriarchy, culture, and socioeconomic equalities were reported.
\end{abstract}

Conclusions: These findings suggest a high burden of DV among young populations, who are vulnerable to various socioecological determinants of DV. Future research should examine how these factors influence DV and multi-level interventions should be adopted to address the same.

Keywords: Dating Violence; Intimate Partner Violence; Umbrella Review; Systematic Review; Meta-analysis

\footnotetext{
${ }^{1}$ Department of Health Promotion and Community Health Sciences, Texas A\&M School of Public Health, College Station, TX 77843, USA.

${ }^{2}$ Nature Study Society of Bangladesh, Khulna 09000, Bangladesh.

${ }^{3}$ IIHMR University, Jaipur, Rajasthan 302029, India.

*Corresponding author: Md Mahbub Hossain, Department of Health Promotion and Community Health Sciences, Texas A\&M School of Public Health, College Station, TX 77843, USA. Email: mhossain@ tamu.edu
} 


\section{Introduction}

Dating violence (DV) is a psychosocial problem affecting the health and wellbeing of many people around the world. The Center for Disease Control and Prevention (CDC) defined DV as a type of intimate partner violence occurring between two people in a close relationship (Center for Disease Control and Prevention (CDC), 2019). Moreover, the United States (US) Department of Justice defined DV as violence committed by an individual who is in a social relationship of a romantic or intimate nature with the victim (The US Department of Justice, n.d.). Similar definitions across different disciplines have conceptualized DV as interpersonal violence including different acts of physical, sexual, or psychological aggression among dating or unmarried romantic partners, who are involved in the mate-selection process (Jouriles, Garrido, Rosenfield, \& McDonald, 2009). Physical forms of DV include use of physical forces to cause any form of harms to the target person in a dating relationship whereas sexual DV include physical force to compel a person to engage in a sexual act, or engaging in sexual act in an abusive manner, or involving the victim in a sexual act without willingness or ability to understand the nature of the act (Breiding, Basile, Smith, Black, \& Mahendra, 2015). Furthermore, psychological or emotional DV involves acts, threats of acts, or coercive tactics, which may include humiliation, control, isolation, or deliberate actions which may affect the mental state of the victim (Breiding et al., 2015; WHO, 2012).

Different studies have revealed a varying prevalence of DV in different populations. A review of studies conducted in the US has found estimates for dating violence perpetration ranging from $26 \%$ to $46 \%$ for physical violence and $3 \%$ to $12 \%$ for sexual violence (Hickman, Jaycox, \& Aronoff, 2004). Furthermore, a 10-year (1999-2009) National Teen DV Trend Study found approximately 1 in 10 female adolescents $(9.9 \%$ to $10.3 \%)$ and 1 in 8 male adolescents $(9.1 \%$ to 
11.8\%) reported experiencing physical DV in the past year (Howard, Debnam, \& Wang, 2013; Howard, Debnam, Wang, \& Gilchrist, n.d.). Moreover, studies from other countries have shown a high burden of DV. A study evaluated DV in samples from the UK and Spain found the prevalence of DV victimization and perpetration as 23\% and 30\%, respectively (Viejo, Monks, Sánchez, \& Ortega-Ruiz, 2016). Another study conducted in Canada found more than $45 \%$ of the study participants reported physical DV (Pedersen \& Thomas, 1992). A high prevalence of DV may have a wide range of negative health consequences. Victims of DV may have physical trauma and a higher risk of HIV and other sexually transmitted diseases (Teitelman, Ratcliffe, Dichter, \& Sullivan, 2008), which may incur high socioeconomic burden. More importantly, DV profoundly impacts the psychosocial processes and mental health of the victims (Temple et al., 2016). Studies have shown high prevalence of psychiatric comorbidities including depression, anxiety, substance abuse, stress disorders, and posttraumatic stress disorder among the victims of DV (Burton, Halpern-Felsher, Rehm, Rankin, \& Humphreys, 2016; Duerksen \& Woodin, 2019; Y. Park, Mulford, \& Blachman-Demner, 2018). The long-term psychosocial outcomes of DV may include fear to new relationship, difficulties in establishing meaningful communication, social isolation, loneliness, and increased risks of subsequent DV victimization and perpetration (Duerksen \& Woodin, 2019; Y. Park et al., 2018).

To address the epidemiological and psychosocial challenges related to DV, it is essential to understand the magnitude of the condition across different population groups. It may help in identifying the high-risk groups and adopt preventive and therapeutic measures to alleviate the burden of DV. Moreover, intrapersonal characteristics, interpersonal relationship dynamics, and sociodemographic factors measures play a critical role in determining the burden of DV in any given population (Foshee et al., 2008). Therefore, it is necessary to examine the determinants of 
DV to better understand the underlying factors associated with the burden of DV across diverse population groups. Several reviews exist which reported either the prevalence or factors associated with DV, but there is no review of the systematic reviews and meta-analyses offering consolidated evidence on prevalence and determinants of DV. Considering there are about 11 systematic reviews published each day, there is a critical need for synthesizing evidence from existing reviews and meta-research to advance knowledge and practice (Aromataris et al., 2015). This umbrella review aims to synthesize the current evidence on the global prevalence and determinants of DV from systematic reviews and meta-analyses, which may inform future scientific, therapeutic, and policy discourses in DV.

\section{Methods}

\section{Searching the Literature}

To conduct this umbrella review, we used the Preferred Reporting Items for Systematic Reviews and Meta-Analyses (PRISMA) guidelines (Liberati et al., 2009) and the Joanna Briggs Institute (JBI) methodology for umbrella reviews (Aromataris et al., 2015). We searched MEDLINE, EMBASE, PubMed, Academic Search Ultimate, CINAHL, PsycINFO, Violence and Abuse Abstracts, Health Source Nursing/Academic Edition, Health Policy Reference Center, and the Cochrane Library to retrieve potential studies relevant to the objective of this umbrella review. To identify systematic reviews and meta-analyses on prevalence and correlates of DV, use used following search syntax: ("date violence" OR "date abuse" OR "date aggression" OR "dating violence" OR "dating abuse" OR "dating aggression" OR "courtship violence" OR "courtship abuse" OR "premarital abuse" OR "premarital violence" OR "premarital aggression") AND (prevalence OR incidence OR statistics OR epidemiology OR predictors OR correlates OR determinants OR "risk factors" OR "associated factors" OR "factors associated" OR factors) 
AND ("systematic review" OR "meta-analysis" OR "pooled estimate" OR "pooled prevalence" OR "pooled effect"). The keywords were used to search titles, abstracts, subject headings (like $\mathrm{MeSH}$ ), and general search terms. No language restriction was applied during database searching. All the databases were searched since the inception of the respective databases. The first search was conducted on August 27, 2019 and same search process was repeated on October 13, 2019, for the last time. In addition to database searching, we retrieved additional articles through hand-searching violence and psychiatry-related journals and communicating with subject-matter experts. Moreover, we used the "cited by" function in Google Scholar to check the eligibility of the citing articles of the primarily screened articles. All the retrieved citations were uploaded to RefWorks and Rayyan software for citation management and systematic evaluation processes. After removing the duplicates, two authors independently evaluated the titles and abstracts of the citations as per the selection criteria of our review. A third author was consulted after the primary screening to confirm the eligibility of the included articles, resolve the conflicts in the selection process, and reach a consensus about the finally recruited articles.

\section{Selection Criteria}

In this umbrella review, we included an article if it: a) was a systematically conducted literature review or research synthesis using qualitative (meta-synthesis) or quantitative (meta-analysis) approaches, b) primarily focused on any type of DV among partners in a dating or romantic relationship, c) synthesized either the prevalence or correlates of DV from the primary studies in respective reviews, d) included full-text in English, e) published as a journal article.

We excluded an article if it: a) was not a systematically conducted review (for example, nonsystematic literature reviews, overviews, primary articles, opinions, letters, editorials were excluded), b) did not primarily focus on any form of DV in dating relationships (for example, 
reviews focusing on domestic or any other violence, or articles presenting dating violence with other types of violence together were excluded), c) did not report either prevalence (including incidence or proportion in a given population, if reported) or determinants (also can be termed as correlates or associated factors in different methods and study designs) of DV synthesized from primary articles, d) did not have the full-text in English, e) was not a journal article (for example, dissertations, policy briefs, reports, book chapters were excluded).

\section{Evaluation of the Methodological Quality of the Recruited Studies}

Critical appraisal of the methodological quality of the recruited reviews is an essential process of conducting an umbrella review (Aromataris et al., 2015). In this review, we evaluated the quality of the systematic reviews and meta-analyses using the JBI critical appraisal checklist for systematic reviews and research synthesis checklist. This checklist facilitates distinguishing the quality of studies and decision-making about the recruitment of the articles. Moreover, this checklist has ten items; each item can receive one point and the overall quality score of a study can range from zero to ten. In this umbrella review, studies receiving zero to four, five to seven, and eight to ten were categorized as low, medium, and high-quality studies, respectively. 

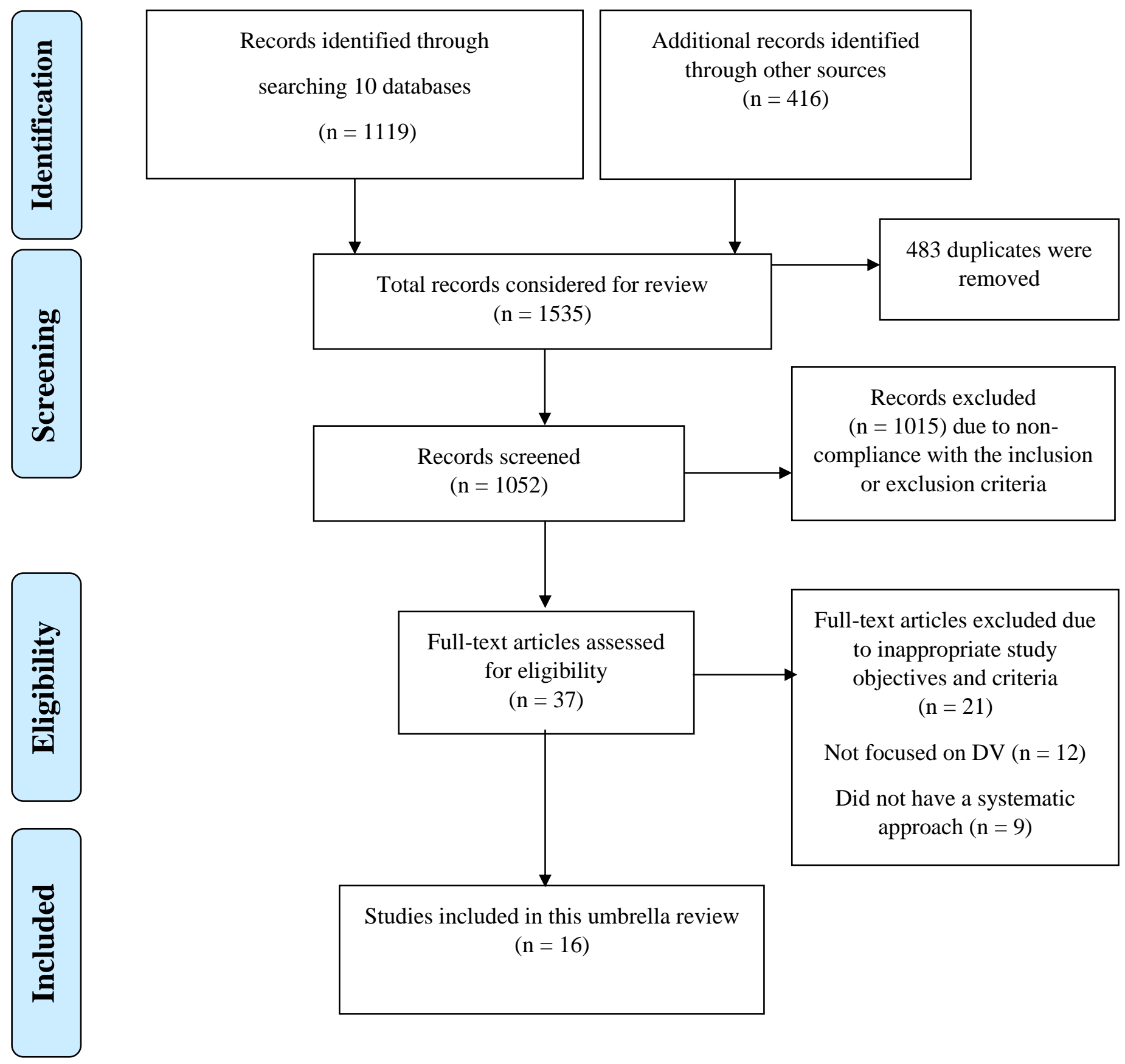

Figure 1: Flow diagram of the review 


\section{Data Extraction and Synthesis}

A codebook for extracting quantitative and qualitative data was prepared using the JBI data extraction tool for systematic reviews and research synthesis (Munn, Tufanaru, \& Aromataris, 2014). Details on the authors, year of publication, characteristics of the review studies, origin and types of the primary studies in each review, sample size and characteristics of the study population, and key findings on prevalence, determinants, correlates, or associated factors of DV were extracted by two authors and reviewed by a third author. The extracted data were narratively synthesized to present the characteristics of the review studies and the findings meeting the objective of this study. Prevalence data were presented as pooled estimates from meta-analysis, as reported in narrative reviews. Moreover, determinants, correlates or factors associated with DV were extracted and evaluated using socioecological model of McLeroy and colleagues (Mcleroy, Bibeau, Steckler, \& Glanz, 1988). Using this approach, different variables associated with DV were categorized as individual, interpersonal, community, social, and systems-level determinants of DV. Qualitative or narrative findings were presented as they were reported in the respective reviews, whereas quantitative data were presented with the range of rates within $95 \%$ confidence interval $(\mathrm{CI})$, wherever reported.

\section{Results}

We found 1119 citations from database searching and 416 citations from additional sources, totaling 1535 citations. After removing the duplicates, screening the titles/abstracts, and evaluating the full texts, we found 16 articles that met our criteria (Caridade, Braga, \& Borrajo, 2019; Duval, Lanning, \& Patterson, 2018; Garthe, Sullivan, \& McDaniel, 2017; Hébert et al., 2019; Johnson et al., 2017; Johnson, Parker, Rinehart, Nail, \& Rothman, 2015; Joly \& Connolly, 2016; S. Park \& Kim, 2019, 2018; Rothman, McNaughton Reyes, Johnson, \& LaValley, 2012; 
Rubio-Garay, López-González, Carrasco, \& Amor, 2017; Spencer, Toews, Anders, \& Emanuels, 2019; Stonard, Bowen, Lawrence, \& Price, 2014; Taquette \& Maia Monteiro, 2019; Wincentak, Connolly, \& Card, 2017; Zych, Viejo, Vila, \& Farrington, 2019). A flow-diagram of the screening process is depicted in Figure 1.

\section{Characteristics of the Studies and Participants}

Among the 16 studies included in this review (summary of the studies is provided in Table 1), ten were meta-analyses (Garthe et al., 2017; Hébert et al., 2019; Johnson et al., 2017; Joly \& Connolly, 2016; S. Park \& Kim, 2018, 2019; Rothman et al., 2012; Spencer et al., 2019;

Wincentak et al., 2017; Zych et al., 2019) and six were systematic reviews (Caridade et al., 2019; Duval et al., 2018; Johnson et al., 2015; Rubio-Garay et al., 2017; Stonard et al., 2014; Taquette \& Maia Monteiro, 2019). These studies were published from 2012 to 2019, while most $(\mathrm{n}=15)$ of the studies were published since 2017. The number of primary studies within those reviews ranged from 13 to 113 , and the sample sizes ranged from 24 to 85,198 . While most participants across all the studies were adolescents, four reviews (Spencer et al., 2019; Taquette \& Maia Monteiro, 2019; Wincentak et al., 2017; Zych et al., 2019) exclusively recruited primary studies with adolescent participants, whereas remaining reviews recruited studies comprising adolescents, young adults, and participants of other age groups. Most primary studies in all the reviews were conducted in the US and Canada; however, four reviews included primary studies conducted in different states of the US only (Duval et al., 2018; Johnson et al., 2017, 2015; Spencer et al., 2019). Very few primary studies were reported from low- and middle-income countries. 
Table 1: Characteristics and the key findings of the included systematic reviews and meta-analyses

\begin{tabular}{|c|c|c|c|c|c|}
\hline Source & $\begin{array}{l}\text { Name and } \\
\text { timeframe of } \\
\text { searching } \\
\text { Databases }\end{array}$ & $\begin{array}{l}\text { Number and } \\
\text { type of } \\
\text { primary } \\
\text { studies }\end{array}$ & $\begin{array}{l}\text { Origin of } \\
\text { included } \\
\text { studies }\end{array}$ & $\begin{array}{l}\text { Sample size and } \\
\text { characteristics of } \\
\text { the study } \\
\text { population (if } \\
\text { reported) }\end{array}$ & $\begin{array}{l}\text { Key findings related to prevalence } \\
\text { and/or associated factors of DV }\end{array}$ \\
\hline $\begin{array}{l}\text { (Rothman } \\
\text { et al., 2012) }\end{array}$ & $\begin{array}{l}\text { PsycINFO, ISI } \\
\text { Web of } \\
\text { Knowledge } \\
\text { (including } \\
\text { citations from } \\
\text { Medline, } \\
\text { BIOSIS, } \\
\text { and other } \\
\text { databases); } \\
\text { 1985-2010 }\end{array}$ & $\begin{array}{l}28(82 \% \\
\text { cross- } \\
\text { sectional })\end{array}$ & $\begin{array}{l}\text { The US }(\mathrm{n}=23), \\
\text { Canada }(\mathrm{n}=1), \\
\text { Mexico }(\mathrm{n}=1), \\
\text { Russia }(\mathrm{n}=1), \\
\text { New Zealand (n } \\
=1), \text { South } \\
\text { Africa }(\mathrm{n}=1)\end{array}$ & $\begin{array}{l}\text { Sample size } \\
\text { ranged from } 40 \text { to } \\
13,422 ; \text { age ranged } \\
\text { from } 12 \text { to } 54 \\
\text { years; } 7 \text { studies } \\
\text { had high school } \\
\text { participants, } 14 \\
\text { had college or } \\
\text { university-based } \\
\text { samples, } 7 \text { had } \\
\text { participants from } \\
\text { communities and } \\
\text { other settings }\end{array}$ & $\begin{array}{l}\text { Odds ratio for DV perpetration for heavy } \\
\text { episodic drinking, problem use, and } \\
\text { frequency/quantity were } 1.47 \text { ( } 95 \% \mathrm{CI} \text { : } \\
1.17 .1 .85), 2.33 \text { (95\% CI: } 1.94,2.8) \text {, } \\
\text { and } 1.23 \text { ( } 95 \% \text { CI: } 1.16,1.31) \text { in fixed- } \\
\text { effect model }\end{array}$ \\
\hline $\begin{array}{l}\text { (Stonard et } \\
\text { al., 2014) }\end{array}$ & $\begin{array}{l}\text { Academic } \\
\text { Search } \\
\text { Complete, } \\
\text { PsycINFO, } \\
\text { Science Direct, } \\
\text { Google Scholar; } \\
\text { Post-2000 } \\
\text { studies }\end{array}$ & $\begin{array}{l}65 \text { ( } 48 \text { cross- } \\
\text { sectional, } 9 \\
\text { longitudinal, } \\
\text { and } 8 \\
\text { retrospective } \\
\text { studies) }\end{array}$ & $\begin{array}{l}\text { United States ( } \mathrm{n} \\
=19), \mathrm{UK}(\mathrm{n}= \\
\text { 17), Canada }(\mathrm{n}= \\
\text { 16), Germany ( } \\
=4) \text { Sweden ( } \mathrm{n} \\
=3 \text { ), Spain }(\mathrm{n}= \\
\text { 2), New Zealand } \\
(\mathrm{n}=2), \\
\text { Netherlands ( } \mathrm{n}= \\
\text { 1), Switzerland } \\
(\mathrm{n}=1)\end{array}$ & $\begin{array}{l}\text { Sample size } \\
\text { ranged from } 117 \\
\text { to } 81247 ; \text { age } \\
\text { ranged from } 10 \text { to } \\
29 \text { years; most } \\
\text { studies recruited } \\
\text { adolescent } \\
\text { participants }\end{array}$ & $\begin{array}{l}12 \% \text { to } 56 \% \text { participants reported some } \\
\text { form of victimization, } 12 \% \text { to } 54 \% \\
\text { reported perpetration of Technology } \\
\text { Assisted Adolescent Dating Violence } \\
\text { and Abuse (TAADVA). Physical } \\
\text { violence victimization (ranged from } 10 \% \\
\text { to } 30 \% \text { ), physical violence perpetration } \\
\text { (5\% to } 30 \% \text { ), psychological or emotional } \\
\text { violence victimization ( } 35 \% \text { to } 55 \% \text { ), } \\
\text { psychological or emotional violence } \\
\text { perpetration ( } 20 \% \text { to } 70 \%) \text {, sexual } \\
\text { violence victimization ( } 5 \% \text { to } 30 \% \text { ), and } \\
\text { sexual violence perpetration ( } 5 \% \text { to } \\
20 \% \text { ) were reported }\end{array}$ \\
\hline
\end{tabular}




\begin{tabular}{|c|c|c|c|c|c|}
\hline $\begin{array}{l}\text { (Johnson et } \\
\text { al., 2015) }\end{array}$ & $\begin{array}{l}\text { PubMed, Web } \\
\text { of Science, } \\
\text { PsycINFO; } \\
2005-2015\end{array}$ & $\begin{array}{l}20 \text { (14 cross- } \\
\text { sectional } \\
\text { studies) }\end{array}$ & $\begin{array}{l}\text { Different states } \\
\text { of the US }\end{array}$ & $\begin{array}{l}11 \text { studies had } \\
\text { male and female } \\
\text { participants, } 9 \text { had } \\
\text { youth from both } \\
\text { urban and non- } \\
\text { urban settings, } 6 \\
\text { focused on } \\
\text { adolescents, } 9 \\
\text { focused on } \\
\text { emerging adults; } \\
15 \text { of the } 20 \\
\text { studies recruited } \\
\text { participants from } \\
\text { school settings }\end{array}$ & $\begin{array}{l}\text { Neighborhood factors associated with } \\
\text { DV included social disorganization ( } 8 \\
\text { studies evaluated association), five } \\
\text { studies reports association with } \\
\text { collective efficacy, neighborhood } \\
\text { disorder (1 out of } 12 \text { studies reported } \\
\text { association with DV), alcohol outlets (4 } \\
\text { studies reported association with DV), } \\
\text { demographic and structural challenges } \\
\text { (11 studies assessed neighborhood-level } \\
\text { structural factors, } 8 \text { investigated } \\
\text { association of residence characteristics } \\
\text { with DV). Neighborhood disorder is } \\
\text { associated with physical dating violence } \\
\text { perpetration, but do not with physical } \\
\text { dating violence victimization. }\end{array}$ \\
\hline $\begin{array}{l}\text { (Joly \& } \\
\text { Connolly, } \\
\text { 2016) }\end{array}$ & $\begin{array}{l}\text { PsycINFO, } \\
\text { ERIC, and } \\
\text { Social Sciences } \\
\text { Abstracts; } \\
\text { timeframe not } \\
\text { specified }\end{array}$ & $\begin{array}{l}29(21 \\
\text { quantitative } \\
\text { and } 8 \\
\text { qualitative } \\
\text { studies })\end{array}$ & $\begin{array}{l}\text { Most studies } \\
\text { were from the } \\
\text { US; } 1 \text { qualitative } \\
\text { study each from } \\
\text { Canada and UK }\end{array}$ & $\begin{array}{l}\text { Sample size } \\
\text { ranged from } 24 \text { to } \\
724 \text { (for } \\
\text { quantitative } \\
\text { studies) and } 1 \text { to } \\
29 \text { (for qualitative } \\
\text { studies); high-risk } \\
\text { population were } \\
\text { surveyed including } \\
\text { street-involved, } \\
\text { pregnant, } \\
\text { maltreated, } \\
\text { juvenile justice } \\
\text { system } \\
\text { participants }\end{array}$ & $\begin{array}{l}34 \% \text { (95\% CI } 24 \% \text { to } 45 \% \text { ) participants } \\
\text { experienced physical DV, } 45 \% \text { (95\% CI } \\
31 \% \text { to } 61 \% \text { ) participants perpetuated } \\
\text { physical DV; factors influencing DV } \\
\text { included vulnerability due to being high- } \\
\text { risk individuals, teenage mothers, } \\
\text { juvenile justice system, truancy, } \\
\text { psychological conditions like } \\
\text { hallucination, delusion, drug use, self- } \\
\text { harm, suicidal ideation, irrational } \\
\text { thinking, and post-traumatic stress } \\
\text { disorder; social factors associated with } \\
\text { DV included negative peer relations, } \\
\text { lack of family and social support, } \\
\text { negative neighborhood experience. }\end{array}$ \\
\hline
\end{tabular}




\begin{tabular}{|c|c|c|c|c|c|}
\hline $\begin{array}{l}\text { (Rubio- } \\
\text { Garay et } \\
\text { al., 2017) }\end{array}$ & $\begin{array}{l}\text { MEDLINE, } \\
\text { PsycINFO, } \\
\text { Academic } \\
\text { Search Premier, } \\
\text { SCOPUS, Web } \\
\text { of Science, and } \\
\text { additional } \\
\text { sources like e- } \\
\text { journals; studies } \\
\text { published till } \\
2013\end{array}$ & $\begin{array}{l}113 \text { (all } \\
\text { quantitative } \\
\text { studies) }\end{array}$ & $\begin{array}{l}\text { Most studies } \\
\text { were conducted } \\
\text { in the US and } \\
\text { other developed } \\
\text { countries }\end{array}$ & $\begin{array}{l}\text { Sample size } \\
\text { ranged from } 490 \\
\text { to } 81247 \text {; most } \\
\text { studies included } \\
\text { adolescents } \\
\text { followed by young } \\
\text { adult and mixed } \\
\text { populations }\end{array}$ & $\begin{array}{l}\text { The prevalence of different forms of DV } \\
\text { was reported as: perpetrated physical } \\
\text { violence ( } 3.8 \% \text { to } 41.9 \%) \text {, victimized } \\
\text { physical violence }(0.4 \% \text { to } 57.3 \%) \text {, } \\
\text { perpetrated psychological violence } \\
\text { ( } 4.2 \% \text { to } 97 \%) \text {, victimized psychological } \\
\text { violence }(8.5 \% \text { to } 95.5 \%) \text {, perpetrated } \\
\text { sexual violence ( } 1.2 \% \text { to } 58.8 \%) \text {, } \\
\text { victimized sexual violence }(0.1 \% \text { to } \\
64.6 \%) \text {. }\end{array}$ \\
\hline $\begin{array}{l}\text { (Garthe et } \\
\text { al., 2017) }\end{array}$ & $\begin{array}{l}\text { PsycINFO, Web } \\
\text { of Science, and } \\
\text { relevant } \\
\text { journals; 2000- } \\
2014\end{array}$ & $\begin{array}{l}27 \text { (all } \\
\text { quantitative } \\
\text { studies) }\end{array}$ & $\begin{array}{l}\text { One study each } \\
\text { was conducted } \\
\text { in Brazil and } \\
\text { Thailand; all the } \\
\text { remaining were } \\
\text { from the US (n } \\
=15) \text { and } \\
\text { Canada }(\mathrm{n}=10)\end{array}$ & $\begin{array}{l}\text { Sample ranged } \\
\text { from } 43 \text { to } 4131 \\
\text { all samples } \\
\text { included } \\
\text { adolescent } \\
\text { participants }\end{array}$ & $\begin{array}{l}\text { Three peer risk factors of DV were meta- } \\
\text { analyzed; peer dating violence }(\mathrm{r}=\cdot 30) \text {, } \\
\text { peers' aggressive and/or antisocial } \\
\text { behavior }(\mathrm{r}=\cdot 20) \text {, and being victimized } \\
\text { by peers }(\mathrm{r}=\cdot 22) \text { were all significantly } \\
\text { related to DV perpetration and } \\
\text { victimization among the adolescents. }\end{array}$ \\
\hline $\begin{array}{l}\text { (Johnson et } \\
\text { al., 2017) }\end{array}$ & $\begin{array}{l}\text { MEDLINE and } \\
\text { PsycINFO; } \\
\text { 2003-2015 }\end{array}$ & $\begin{array}{l}13 \text { ( } 7 \text { cross- } \\
\text { sectional, } 5 \\
\text { longitudinal, } \\
1 \\
\text { retrospective) }\end{array}$ & $\begin{array}{l}\text { Different states } \\
\text { of the US }\end{array}$ & $\begin{array}{l}\text { Sample size } \\
\text { ranged from } 67 \text { to } \\
15123 \text {; } \\
\text { participants were } \\
\text { adolescents and } \\
\text { emerging adults }\end{array}$ & $\begin{array}{l}\text { Marijuana use was associated with } \\
\text { physical DV victimization (OR } 1.54 \text {, } \\
95 \% \text { CI: } 1.22-1.93 \text { ) and perpetration (OR } \\
1.45,95 \% \text { CI: } 1.2-1.75)\end{array}$ \\
\hline $\begin{array}{l}\text { (Wincentak } \\
\text { et al., 2017) }\end{array}$ & $\begin{array}{l}\text { PsycINFO, } \\
\text { Sociological } \\
\text { Abstracts, and } \\
\text { the Education } \\
\text { Resources }\end{array}$ & $\begin{array}{l}101 \text { (all } \\
\text { quantitative) }\end{array}$ & Not specified & $\begin{array}{l}\text { Sample size } \\
\text { ranged from } 48 \text { to } \\
81247 ; \\
\text { participants aged } \\
13 \text { to } 18 \text { years }\end{array}$ & $\begin{array}{l}\text { The overall prevalence of physical and } \\
\text { sexual DV was } 20 \% \text { (95\% CI: } 17 \%- \\
23 \% \text { ) and } 9 \% \text { (95\% CI: } 5 \%-14 \% \text { ) } \\
\text { respectively with significant variability } \\
\text { across studies; physical DV ranged from } \\
1 \% \text { to } 61 \% \text {, and sexual DV ranged from } \\
\text { less than } 1 \% \text { to } 54 \% \text {. Boys and girls }\end{array}$ \\
\hline
\end{tabular}




\begin{tabular}{|c|c|c|c|c|c|}
\hline & $\begin{array}{l}\text { Information } \\
\text { Center (ERIC); } \\
1980-2013\end{array}$ & & & & $\begin{array}{l}\text { experienced similar rate }(21 \%) \text { of } \\
\text { victimization; however, boys } \\
\text { experienced less }(13 \%) \text { perpetration } \\
\text { compared to girls }(25 \%) \text {. Girls reported } \\
\text { lower rates of perpetration compared } \\
\text { with boys ( } 3 \% \text { vs } 10 \%) \text { and higher rates } \\
\text { of victimization ( } 14 \% \text { vs } 8 \%) \text {. Sample } \\
\text { groups with higher prevalence had older } \\
\text { teens (sexual DV), cultural minority girls } \\
\text { (physical DV), and disadvantaged } \\
\text { neighborhoods (physical DV) }\end{array}$ \\
\hline $\begin{array}{l}\text { (Duval et } \\
\text { al., 2018) }\end{array}$ & $\begin{array}{l}\text { EBSCO, } \\
\text { Scopus, } \\
\text { PsycINFO, } \\
\text { MEDLINE, } \\
\text { ERIC, and } \\
\text { PubMed; 2006- } \\
2016\end{array}$ & $\begin{array}{l}23(22 \\
\text { quantitative, } \\
1 \text { mixed } \\
\text { method) }\end{array}$ & $\begin{array}{l}\text { Different states } \\
\text { of the US }\end{array}$ & $\begin{array}{l}\text { Sample size } \\
\text { ranged from } 43 \text { to } \\
5035 \text {, aged } \\
\text { between } 18 \text { to } \\
22.06 \text { years; } \\
\text { undergraduate } \\
\text { students from } \\
\text { different academic } \\
\text { institutions }\end{array}$ & $\begin{array}{l}\text { Female participants were more likely to } \\
\text { perpetrate and/or experience DV than } \\
\text { males. Up to } 43.3 \% \text { males perpetrated } \\
\text { DV whereas up to } 50.4 \% \text { females were } \\
\text { victims of DV. Individual risk factors } \\
\text { included substance use, high-risk sexual } \\
\text { behaviors; family risk factors included } \\
\text { intergenerational violence; and peer and } \\
\text { social risk factors included Greeklife or } \\
\text { athletic team membership. Self-control } \\
\text { and positive relationship type were } \\
\text { reported as protective factors. }\end{array}$ \\
\hline $\begin{array}{l}\text { (S. Park \& } \\
\text { Kim, 2018) }\end{array}$ & $\begin{array}{l}\text { PubMed, Web } \\
\text { of Science, and } \\
\text { SCOPUS; } \\
\text { studies } \\
\text { published till } \\
2016\end{array}$ & $\begin{array}{l}27 \text { (all } \\
\text { quantitative } \\
\text { studies) }\end{array}$ & $\begin{array}{l}\text { The US }(n=19) \text {, } \\
\text { Canada }(n=6), \\
\text { Taiwan }(n=1), \\
\text { Switzerland }(n= \\
\text { 1) }\end{array}$ & $\begin{array}{l}15 \text { studies } \\
\text { included teenagers } \\
\text { and } 12 \text { studies } \\
\text { included adults; } \\
\text { age ranged from } \\
11 \text { to } 21.5\end{array}$ & $\begin{array}{l}\text { Correlates of DV included family related } \\
\text { factors like family structure and } \\
\text { relationship, parenting, witnessing IPV, } \\
\text { childhood maltreatment, fear of family } \\
\text { violence, parents' education, } \\
\text { socioeconomic status. Community } \\
\text { related correlates are bullying, deviant } \\
\text { peers, positive friendship, grade, school } \\
\text { attachment, neighborhood hazard and } \\
\text { support. Having deviant peers was the }\end{array}$ \\
\hline
\end{tabular}




\begin{tabular}{|c|c|c|c|c|c|}
\hline & & & & & $\begin{array}{l}\text { strongest risk factor of DV perpetration, } \\
\text { whereas witnessing parental violence } \\
\text { was the strongest risk factor of DV } \\
\text { victimization. Also, risk factors of DV } \\
\text { were more powerful predictors of DV } \\
\text { perpetrators and victims than the } \\
\text { protective factors. }\end{array}$ \\
\hline $\begin{array}{l}\text { (Caridade } \\
\text { et al., 2019) }\end{array}$ & $\begin{array}{l}\text { Academic } \\
\text { Research } \\
\text { Complete, } \\
\text { Business Source } \\
\text { Complete, } \\
\text { Complementary } \\
\text { Index, ERIC/ } \\
\text { EBSCOhost, } \\
\text { Psychology and } \\
\text { Behavioral } \\
\text { Sciences } \\
\text { Collection, } \\
\text { PubMed, } \\
\text { Science Direct, } \\
\text { SCOPUS, and } \\
\text { Social Sciences } \\
\text { Citation Index; } \\
\text { studies } \\
\text { published till } \\
\text { 2018 }\end{array}$ & $\begin{array}{l}44 \text { (most } \\
\text { studies were } \\
\text { cross- } \\
\text { sectional) }\end{array}$ & $\begin{array}{l}\text { Spain }(\mathrm{n}=8), \\
\text { Mexico }(\mathrm{n}=3) \text {, } \\
\text { one study each } \\
\text { from Belgium, } \\
\text { Israel, Italy, } \\
\text { Canada, UK, } \\
\text { and Portugal; } \\
\text { remaining } \\
\text { studies from the } \\
\text { US }\end{array}$ & $\begin{array}{l}\text { Sample size } \\
\text { ranged from } 155 \\
\text { to } 5647 \text {, age } \\
\text { ranged from } 12 \text { to } \\
25 \text { years; most } \\
\text { studies recruited } \\
\text { students from } \\
\text { academic and } \\
\text { community } \\
\text { settings }\end{array}$ & $\begin{array}{l}\text { Prevalence of cyber DV perpetration } \\
\text { ranged from } 8.1 \% \text { to } 93.7 \% \text {; prevalence } \\
\text { of DV victimization ranged from } 5.8 \% \\
\text { to } 92 \% \text {; cyber DA perpetration was } \\
\text { related to legitimization of DV by boys, } \\
\text { behavioral or romantic jealousy, beliefs } \\
\text { in myths of love, sexist beliefs, } \\
\text { endorsement of gender stereotypes, } \\
\text { narcissism vulnerability and grandiosity, } \\
\text { experiencing other forms of violence, } \\
\text { offline DV experience, family violence, } \\
\text { bullying and cyber bullying; cyber } \\
\text { victimization was related to delinquency } \\
\text { or antisocial behaviors, other high-risk } \\
\text { behavior, initiated sexual activity, time } \\
\text { spent on social networking, higher } \\
\text { anxiety or depressive symptoms, other } \\
\text { psychological issues, sexual orientation, } \\
\text { worse dyadic adjustment. }\end{array}$ \\
\hline $\begin{array}{l}\text { (Hébert et } \\
\text { al., 2019) }\end{array}$ & $\begin{array}{l}\text { PsycINFO, } \\
\text { PubMed, } \\
\text { Google Scholar, } \\
\text { and relevant } \\
\text { French }\end{array}$ & $\begin{array}{l}87 ; \text { study } \\
\text { types were } \\
\text { not reported }\end{array}$ & $\begin{array}{l}\text { The US }(72 \%) \text {, } \\
\text { Canada }(19 \%) \text {, } \\
\text { other studies } \\
\text { from Asia } \\
\text { (South Korea }\end{array}$ & $\begin{array}{l}76 \text { studies } \\
\text { included in meta- } \\
\text { analysis had a total } \\
\text { sample was } \\
278,712 \\
\text { participants; }\end{array}$ & $\begin{array}{l}\text { Peer risk factors included peer } \\
\text { victimization }(\mathrm{r}=\cdot 186) \text {, peer sexual } \\
\text { harassment }(\mathrm{r}=\cdot 297) \text {, deviant peers }(\mathrm{r}= \\
\cdot 25) \text {; protective factors included parental } \\
\text { monitoring }(\mathrm{r}=-\cdot 126) \text {, parental support } \\
(\mathrm{r}=-\cdot 109) \text {, peer support }(\mathrm{r}=-\cdot 139)\end{array}$ \\
\hline
\end{tabular}




\begin{tabular}{|c|c|c|c|c|c|}
\hline & $\begin{array}{l}\text { databases; 2000- } \\
2015\end{array}$ & & $\begin{array}{l}\text { and India), } \\
\text { Europe } \\
\text { (Spain), and } \\
\text { South America } \\
\text { (Mexico, Chile, } \\
\text { and El Salvador) }\end{array}$ & $\begin{array}{l}\text { sample ranged } \\
\text { from } 41 \text { to } 85,198 ; \\
62 \% \text { samples } \\
\text { included } \\
\text { adolescents and } \\
33 \% \text { had emerging } \\
\text { adult participants }\end{array}$ & \\
\hline $\begin{array}{l}\text { (S. Park \& } \\
\text { Kim, 2019) }\end{array}$ & $\begin{array}{l}\text { PubMed, Web } \\
\text { of Science, and } \\
\text { SCOPUS; } \\
\text { studies } \\
\text { published till } \\
2016\end{array}$ & $\begin{array}{l}25 \text { (all } \\
\text { quantitative } \\
\text { studies) }\end{array}$ & $\begin{array}{l}\text { Place not } \\
\text { specified }\end{array}$ & $\begin{array}{l}\text { Mean age of the } \\
\text { participants ranged } \\
\text { from } 13.8 \text { to } 23 ; \\
\text { most participants } \\
\text { were adolescents } \\
\text { or young adults }\end{array}$ & $\begin{array}{l}\text { DV victims and perpetrators were more } \\
\text { likely to be involved in violence } \\
\text { experiences. DV victims or DV } \\
\text { perpetrators were more likely to assume } \\
\text { the opposite role as well. Also, DV } \\
\text { perpetrators had a strong association } \\
\text { with previous or concurrent experience } \\
\text { of DV victimization, and current DV } \\
\text { victims had experiences of being both } \\
\text { victim and perpetrator. DV victimization } \\
\text { had a stronger association with general } \\
\text { violence experiences than did DV } \\
\text { perpetration }\end{array}$ \\
\hline $\begin{array}{l}\text { (Spencer et } \\
\text { al., 2019) }\end{array}$ & $\begin{array}{l}\text { PsycINFO, } \\
\text { PubMed, } \\
\text { Proquest, } \\
\text { Proquest } \\
\text { Dissertations } \\
\text { and Theses, } \\
\text { Education } \\
\text { Resources } \\
\text { Information } \\
\text { Center (ERIC), } \\
\text { and Social } \\
\text { Services }\end{array}$ & $\begin{array}{l}37 \text { ( } 20 \\
\text { longitudinal, } \\
17 \text { cross- } \\
\text { sectional) }\end{array}$ & $\begin{array}{l}\text { Different states } \\
\text { of the US }\end{array}$ & $\begin{array}{l}\text { Sample size } \\
\text { included in the } \\
\text { meta-analysis was } \\
\text { 33,537; all studies } \\
\text { recruited } \\
\text { adolescent } \\
\text { participants }\end{array}$ & $\begin{array}{l}\text { Physical DV victimization was the } \\
\text { strongest risk marker for physical DV } \\
\text { perpetration. Also, externalizing } \\
\text { behaviors, anger, witnessing violence, } \\
\text { approval of violence, risky sexual } \\
\text { behaviors, controlling behavior, } \\
\text { substance abuse, depression, childhood } \\
\text { maltreatment and delinquency were the } \\
\text { strongest risk markers for DV } \\
\text { perpetration. In contrast, conflict } \\
\text { resolution skills, relationship quality } \\
\text { with parents, and responsibility were } \\
\text { protective markers against TDV }\end{array}$ \\
\hline
\end{tabular}




\begin{tabular}{|c|c|c|c|c|c|}
\hline & $\begin{array}{l}\text { Abstracts; 1997- } \\
2018\end{array}$ & & & & $\begin{array}{l}\text { perpetration. DV perpetration and } \\
\text { depression were significantly stronger } \\
\text { risk markers for female perpetration } \\
\text { whereas controlling behaviors was a } \\
\text { significantly stronger risk marker for } \\
\text { male TDV perpetration }\end{array}$ \\
\hline $\begin{array}{l}\text { (Taquette } \\
\text { \& Maia } \\
\text { Monteiro, } \\
\text { 2019) }\end{array}$ & $\begin{array}{l}\text { PubMed; studies } \\
\text { published till } \\
2017\end{array}$ & $\begin{array}{l}35 \text { ( } 25 \text { cross- } \\
\text { sectional, } 9 \\
\text { longitudinal, } \\
1 \text { qualitative) }\end{array}$ & $\begin{array}{l}\text { The US }(\mathrm{n}=25) \text {, } \\
\text { Spain }(\mathrm{n}=2), \\
\text { South Africa ( } \mathrm{n} \\
=2) \text {, and one } \\
\text { study each in } \\
\text { Canada, Egypt, } \\
\text { Liberia, Malawi, } \\
\text { Rwanda, } \\
\text { Zambia, } \\
\text { Sweden, and the } \\
\text { UK }\end{array}$ & $\begin{array}{l}\text { Sample size } \\
\text { ranged from } 39 \text { to } \\
75590 ; \text { all } \\
\text { participants were } \\
\text { adolescents }\end{array}$ & $\begin{array}{l}\text { Varying prevalence of DV was reported } \\
\text { across studies (up to } 70.7 \% \text { ), factors } \\
\text { associated with DV included patriarchal } \\
\text { culture, racism, heterosexism, poverty, } \\
\text { contextual issues in family, school, } \\
\text { community and social media, health } \\
\text { problems including depression, anxiety, } \\
\text { low self-esteem, substance abuse, and } \\
\text { high-risk sexual behavior }\end{array}$ \\
\hline $\begin{array}{l}\text { (Zych et al., } \\
\text { 2019) }\end{array}$ & $\begin{array}{l}\text { Web of Science, } \\
\text { Google Scholar, } \\
\text { SCOPUS; } \\
\text { studies } \\
\text { published till } \\
2016\end{array}$ & $\begin{array}{l}23 \text { (all } \\
\text { quantitative } \\
\text { studies) }\end{array}$ & $\begin{array}{l}\text { The US }(n=15), \\
\text { Canada }(n=6), \\
\text { one study each } \\
\text { from Norway } \\
\text { and South } \\
\text { Africa }\end{array}$ & $\begin{array}{l}\text { Sample size } \\
\text { ranged from } 88 \text { to } \\
17,780 ; \text { all } \\
\text { participants were } \\
\text { adolescents }\end{array}$ & $\begin{array}{l}\text { DV perpetration was associated with } \\
\text { bullying perpetration (AOR }=1.29,95 \% \\
\text { CI: } 1.07-1.56) \text {, DV victimization was } \\
\text { associated with bullying perpetration } \\
\text { (AOR }=1.09,95 \% \text { CI: } 1.01-1.12 \text { ), which } \\
\text { was stronger for males. Bullying } \\
\text { victimization was related to DV } \\
\text { victimization (AOR }=1.96,95 \% \mathrm{CI} \text { : } \\
\text { 1.68-2.29), which was stronger for } \\
\text { females. The relation between bullying } \\
\text { victimization and DV perpetration was } \\
\text { not statistically significant (AOR = } 1.01 \text {, } \\
95 \% \text { CI: } 0.99-1.03 \text { ) }\end{array}$ \\
\hline
\end{tabular}


For example, among 87 studies included in a meta-analysis by He'bert and colleagues, only two primary studies were from India and El Salvador (Hébert et al., 2019). Most of the studies ( $\mathrm{n}=$ 11) (Duval et al., 2018; Garthe et al., 2017; Hébert et al., 2019; Johnson et al., 2017; S. Park \& Kim, 2018, 2019; Rothman et al., 2012; Spencer et al., 2019; Stonard et al., 2014; Wincentak et al., 2017; Zych et al., 2019) included in this umbrella review had high quality whereas remaining ( $n=5$ ) (Caridade et al., 2019; Johnson et al., 2015; Joly \& Connolly, 2016; Rubio-Garay et al., 2017; Taquette \& Maia Monteiro, 2019) studies had medium quality as per the JBI quality appraisal checklist (Appendix 1).

\section{Prevalence of DV}

Physical DV. Studies have reported a varying prevalence of physical DV. Stonard and colleagues reported the prevalence ranging from 5\% to $30 \%$ and $10 \%$ to $30 \%$ for physical DV perpetration and victimization, respectively (Stonard et al., 2014). Another systematic review by Rubio-Garay and colleagues reported the rates of physical DV perpetration and victimization, ranging from $3.8 \%$ to $41.9 \%$ and $0.4 \%$ to $57.5 \%$, respectively (Rubio-Garay et al., 2017). Two meta-analyses reported the pooled prevalence of Physical DV (Joly \& Connolly, 2016; Wincentak et al., 2017). In the meta-analysis by Joly and colleagues, 34\% (95\% CI: 24\%-45\%) participants experienced physical DV, whereas 45\% (95\% CI: 31\%-61\%) participants perpetrated physical violence in their dating relationships (Joly \& Connolly, 2016). Another meta-analysis of 96 studies found the overall prevalence of reported physical DV was 20\% (95\% CI: 17\%-23\%) (Wincentak et al., 2017).

Sexual DV. A meta-analysis by Wincentak and colleagues found the prevalence of sexual DV as 9\% (95\% CI: 5\%-14\%) (Wincentak et al., 2017). Moreover, Duval and colleagues reported $43.3 \%$ of men claimed to perpetrate sexual DV, whereas $50.4 \%$ of women reported being a 
victim of sexual DV (Duval et al., 2018). Stonard and colleagues found the rates of sexual DV perpetration and victimization ranging from 5\% to $20 \%$ and 5\% to 30\% respectively (Stonard et al., 2014). Furthermore, Rubio-Garay and colleagues reported $1.2 \%$ to $58.8 \%$ of participants perpetrated sexual DV whereas $0.1 \%$ to $64.6 \%$ reported being victims of sexual DV (RubioGaray et al., 2017).

Psychological and Emotional DV. Studies have also evaluated the prevalence of psychological and emotional DV (Rubio-Garay et al., 2017; Stonard et al., 2014). Stonard and colleagues found $35 \%$ to $55 \%$ of participants reported psychological or emotional DV victimization whereas $20 \%$ to $70 \%$ reported perpetration of such violence (Stonard et al., 2014). Another review by RubioGaray and colleagues revealed the prevalence of psychological DV victimization and perpetration ranging from $8.5 \%$ to $95.5 \%$ and $4.2 \%$ to $97 \%$ respectively (Rubio-Garay et al., 2017).

Other Forms of DV. In addition to the above-mentioned typologies, DV is also reported to happen in digital platforms. Stonard and colleagues found the prevalence of technology-assisted DV perpetration ranged from $12 \%$ to $54 \%$ whereas victimization ranged from $12 \%$ to $56 \%$ among the study participants (Stonard et al., 2014). DV in the digital era was also evaluated by Caridade and colleagues who found the prevalence of cyber dating abuse perpetration ranging from $8.1 \%$ to $93.7 \%$ and victimization ranging from $5.8 \%$ to $92 \%$ (Caridade et al., 2019).

\section{Determinants of DV}

Individual-level determinants. Several determinants of DV were reported across studies that may influence the perpetration or victimization of DV among the individuals. Personality and behavioral attributes like low esteem (Taquette \& Maia Monteiro, 2019), bullying (Caridade et 
al., 2019; Zych et al., 2019), narcissism vulnerability (Caridade et al., 2019), grandiosity (Caridade et al., 2019), and controlling behavior (Spencer et al., 2019) were found to be associated with DV. Moreover, alcoholism and other forms of substance abuse were associated with DV (Caridade et al., 2019; Duval et al., 2018; Johnson et al., 2017, 2015; Joly \& Connolly, 2016; Rothman et al., 2012; Spencer et al., 2019; Taquette \& Maia Monteiro, 2019). For example, a meta-analysis by Rothman and colleagues reported higher odds ratio (OR) of DV perpetration for heavy episodic drinking (OR 1.47, 95\%CI: 1.17-1.85), problematic use (OR 2.33, 95\%CI: 1.94-2.8), and frequency/quantity of drinking (OR 1.23, 95\%CI: 1.16-1.31) (Rothman et al., 2012). Furthermore, psychiatric disorders like depression, suicidal ideation, and posttraumatic stress disorder were associated with DV perpetration and victimization (Caridade et al., 2019; Joly \& Connolly, 2016; Spencer et al., 2019; Taquette \& Maia Monteiro, 2019). In addition, poor educational attainment (S. Park \& Kim, 2018), high-risk sexual behavior (Caridade et al., 2019; Duval et al., 2018; Spencer et al., 2019; Taquette \& Maia Monteiro, 2019), and belonging to vulnerable groups like teenage mothers (Joly \& Connolly, 2016) were associated with DV. Also, previous exposure to any forms of violence, including childhood abuse or domestic violence, was associated with DV perpetration and victimization (Caridade et al., 2019; Duval et al., 2018; S. Park \& Kim, 2018, 2019; Spencer et al., 2019; Zych et al., 2019). For example, a meta-analysis by Park and Kim revealed that participants who experienced violence earlier were more likely to perpetrate or become the victims of DV (S. Park \& Kim, 2019). While most of the determinants were reported as risk factors for DV, individual characteristics like self-control (Hébert et al., 2019) and conflict resolution skills (Spencer et al., 2019) have been reported as protective factors against DV. 
Interpersonal determinants. Interpersonal relationships and interactions have been considered as critical elements in DV studies. The characteristics of the peers and the quality of interactions with them are found as major determinants of DV (Duval et al., 2018; Garthe et al., 2017; Hébert et al., 2019; Joly \& Connolly, 2016; S. Park \& Kim, 2018, 2019; Spencer et al., 2019; Zych et al., 2019). For example, a meta-analysis by Garthe and colleagues reported peers' aggressive or antisocial behavior $(r=.20)$, peer DV $(r=.30)$, and being victimized by peers $(r=.22)$ were significantly associated with DV perpetration and victimization (Garthe et al., 2017). Moreover, family structure and relationships were associated with DV among children, adolescents, and young adults (Joly \& Connolly, 2016; S. Park \& Kim, 2018; Spencer et al., 2019; Taquette \& Maia Monteiro, 2019). For example, the meta-analysis by Park and Kim found that several family factors predicted DV, among which "witnessing parental violence" was the strongest risk factor of DV victimization (S. Park \& Kim, 2018). In contrast, parent support (Hébert et al., 2019) and relationship quality with parents (Spencer et al., 2019) were protective factors against DV.

Community-level level determinants. Studies found that community and neighborhood characteristics often influence DV perpetration and victimization (Johnson et al., 2015; Joly \& Connolly, 2016; Wincentak et al., 2017). Johnson and colleagues found neighborhood disorder, disorganization, demographic and structural challenges, and residence characteristics were associated with DV perpetration but not with victimization (Johnson et al., 2015). A metaanalysis Joly and colleagues (Joly \& Connolly, 2016) reported negative neighborhood experience was associated with DV whereas samples from disadvantaged neighborhoods had higher physical DV is a meta-analysis conducted by Wincentak and colleagues (Wincentak et al., 2017). 
Social and systems-level determinants. Several social and systems-level determinants of DV were reported across studies. Social norms like patriarchal social structure (Taquette \& Maia Monteiro, 2019) and legitimacy of DV by boys (Caridade et al., 2019) were associated with DV. Moreover, social structures, institutions, and practices were associated with power imbalance and vulnerability to DV (Johnson et al., 2015; Joly \& Connolly, 2016; Taquette \& Maia Monteiro, 2019; Wincentak et al., 2017). For example, moderator analyses in the meta-analysis by Wincentak and colleagues found samples with socioeconomically disadvantaged and cultural minority girls had higher rates of physical DV (Wincentak et al., 2017). Moreover, Joly and colleagues found participants in the juvenile justice system were more vulnerable to DV (Joly \& Connolly, 2016). Furthermore, Taquette and colleagues reported social and systems-level determinants like culture, racism, sexism, and poverty were associated with DV perpetration and victimization (Taquette \& Maia Monteiro, 2019).

\section{Discussion}

This umbrella review evaluated the current evidence on the prevalence and determinants of DV from published systematic reviews and meta-analyses. The findings suggest a high burden of different forms of DV perpetration and victimization across different population groups. In addition, the determinants of DV at different levels of the socioecological model inform how individual, interpersonal, community, social and systems-level factors may influence DV among the study participants. These findings offer several critical insights about DV, which are essential for future research, policy development, and practice. 
First, the reported prevalence of DV may provide a sub-optimal picture of the actual burden. A possible reason for this may be under-reporting due to stigma and other psychosocial barriers perceived by the affected individuals (Foshee et al., 2008; Hickman et al., 2004; Temple et al., 2016). Healthcare professionals, social workers, legal service providers, and other stakeholders in health and social care should provide support and create an enabling environment that helps the victims to report the cases of DV (Hickman et al., 2004; Y. Park et al., 2018). Another reason may be attributable to the definitions and constructs of DV across studies published from different disciplines. Differences in operational definitions of DV and methods to measure the same may contribute to heterogeneity in estimating the problems and factors associated with DV (Stonard et al., 2014). Future scientific discourses should standardize the definitions and methods, which may facilitate more accurate estimations of the prevalence and determinants of DV.

Second, most of the study participants in the primary studies of the included reviews were adolescents and young adults. A high prevalence of DV among this young population illustrates profound mental health implications. Moreover, DV was found to be associated with behavioral abnormalities, alcoholism and substance abuse, and several psychiatric comorbidities (Burton et al., 2016; Duerksen \& Woodin, 2019; Y. Park et al., 2018; Taquette \& Maia Monteiro, 2019). This may create a complex scenario of psychiatric multimorbidity among individuals who perpetrate or experience DV. Identifying such cases and addressing the same with multipronged mental health services can be difficult. Mental health researchers and practitioners may consider future research and collaborative actions to better understand such psychosocial complexities associated with DV, which may help in understanding and managing such problems. 
Third, most primary studies in this review were conducted in the US, Canada, and countries with similar socioeconomic contexts. A lack of published studies from resource-constrained countries indicates substantial gaps in the research activities or publications in this domain. This appears to be paradoxical as many low- and middle-income countries have a high burden of child marriage and gender-based violence. It is difficult to say whether the concept of dating violence is not yet established in those contexts, or the dominant paradigm of violence research is concentrated on gender-based violence in those countries. However, very few studies from developing countries have shown a similar burden of DV in those contexts highlighting prospects for future research. Moreover, with demographic and epidemiological transitions in those countries, many people are having increased life expectancy and getting married at a later age, which is contributing to a reduction in child marriage in many countries (Das Gupta et al., 2014). Therefore, a larger proportion of the adolescent and young adult population who are unmarried, may get involved in dating relationships. Given the existence of the determinants of DV revealed in this review, those individuals may experience DV in their dating relationships, which is essential to be measured, prevented, and treated, whenever it occurs.

Fourth, the emergence of technology-assisted or cyber DV in recent years is a major mental health concern. With the rapidly increasing number of people using gadgets and having access to the internet may contribute to the growing burden of such violence among people who share the psycho-epidemiological characteristics of DV perpetration or victimization. The similarities and differences of such cases with other forms of DV may help in understanding the phenomenology of DV in the era of digital technologies and address the same. Moreover, higher access to technologies also offers unique opportunities to use such platforms for delivering interventions promoting awareness and preventing DV (Draucker et al., 2019; O'Brien, Sauber, Kearney, 
Venaglia, \& Lemay, 2019), which should be evaluated before large-scale adoption of such approaches.

Lastly, different determinants of DV at multiple levels of the socioecological model inform the complexities among the levels and how they affect DV among the individuals. Risk factors identified at the individual level can help in improving the existing guidelines and approaches to diagnose or predict the potential cases of DV. Moreover, individual characteristics may be helpful in designing more personalized care for DV-affected people. Also, interpersonal factors like peer and family relationships highlight the role of dyadic interactions on the psychosocial wellbeing of the individuals. It is essential to consider critical factors like deviant peers or negative family relationships in DV research and practice to alleviate the burden of DV. In addition, community-level characteristics like neighborhood problems may necessitate a multisectoral and participatory approach involving community members, planners, social workers, development organizations, and other stakeholders to identify the prevalent causes of DV in the community settings. Furthermore, social and systems-level determinants inform the macroenvironmental attributes of DV, which should be addressed in a collective manner. For example, educational institutions are part of the social system, which may play critical roles in delivering interventions preventing DV (Carlos et al., 2017). Such opportunities should be explored, and future interventions should incorporate behavior change constructs considering diverse social and institutional factors, which may increase awareness about DV and prevent the same at the population-level. Nevertheless, social challenges including racism, sexism, impaired health rights in juvenile justice systems, and structural inequalities should be carefully examined and addressed at policy level to prevent the DV epidemic, as those issues continue to affect the mental state of people. 
This review has several limitations, which should be acknowledged. Although we searched major databases, but there may be additional literature which we could not evaluate in this review. Another limitation can be the publication bias. Systematic reviews and meta-analyses with insignificant findings may not have been published, which remain beyond the scope of our review. Moreover, we included studies that explicitly focused on DV. Studies focusing on domestic or gender-based or intimate partner violence were excluded from this review. Those studies may have included DV-related information, which may have provided different insights into this area. Furthermore, we categorized the determinants using the socioecological model. In this process, one determinant may have appeared at multiple levels. For example, previous experience of violence was reported at the individual level, whereas violence at peer or family relationships was reported at the interpersonal level. Such overarching and interconnected factors should be deeply investigated in case of DV to address the problems. Lastly, we did not compare individual primary studies and kept the scope of our review within the reviews and metaanalyses. An in-depth and different organization of individual studies may have considered the in-between studies offering different understanding than an umbrella review approach. However, we conducted this review using state-of-the-art guidelines, and each step of the review process was conducted by more than two authors to minimize biases. Future research should address our limitations and advance the evidence base on DV.

\section{Conclusion}

This umbrella review synthesized the current evidence on the prevalence and determinants of DV from systematic reviews and meta-analyses. This evidence provides an overall understanding of DV among the study populations. A high prevalence and various socioecological determinants 
of DV may inform the development and implementation of policies and interventions to address DV across populations. Also, the research gaps found in the existing literature may inform prospective measures to advance the existing knowledge base. The collective insights of this umbrella review should be used to promote better research and evidence-based approaches preventing DV among people who are or will be in dating relationships.

\section{Critical findings of this umbrella review:}

1. This review identified 16 eligible systematic reviews and meta-analyses reporting the prevalence and determinant of DV.

2. In the included reviews, most of the primary studies were conducted in developed countries, and the majority of the participants were adolescents and young adults

3. Studies reported varying prevalence of physical, sexual, psychological/emotional, and technology-assisted DV ranging from $0.1 \%$ to $57.5 \%, 0.1 \%$ to $64.6 \%, 4.2 \%$ to $97 \%$, and $5.8 \%$ to $92 \%$, respectively.

4. The socioecological determinants of DV included individual behavior, substance abuse, psychiatric conditions, experiencing violence and maltreatment; interpersonal factors like family and peer relationships; community and neighborhood characteristics; patriarchy, culture, and socioeconomic equalities. 


\section{Implications on Practice, Research, and Policy}

To the best of our knowledge, this umbrella review is the first one to examine the consolidated evidence on the prevalence and determinants of DV. This review found a high prevalence and diverse socioecological determinants of DV, which may have following implications for practice, research, and policy:

1. Evidence synthesized in this review provide insights on the severity and determinants of DV, which inform how different populations at-risk should be targeted for evidencebased preventive measures.

2. An understanding of socioecological determinants may allow the development and implementation of interventions improving behavioral and psychiatric conditions of the individuals, and enhancing the nature and quality of family and peer relationships

3. Policies and programs should be adopted to improve the neighborhood characteristics, address cultural disparities, and alleviate social inequalities, which may contribute to a long-term prevention of DV in a given population.

4. The gaps in the existing literature and limitations of this review necessitate future studies and evidence synthesis to better understand DV and associated factors in diverse population group to strengthen the evidence base and inform better policies and practices.

Acknowledgement: None

Funding: No funding was received at any stage of conducting this review

Conflicts of interest: We declare no conflicts of interest 


\section{References}

Aromataris, E., Fernandez, R., Godfrey, C. M., Holly, C., Khalil, H., \& Tungpunkom, P. (2015). Summarizing systematic reviews: methodological development, conduct and reporting of an umbrella review approach review of reviews, systematic review, umbrella review, umbrella review methodology. International Journal of Evidence-Based Healthcare. https://doi.org/10.1097/XEB.0000000000000055

Breiding, M. J., Basile, K. C., Smith, S. G., Black, M. C., \& Mahendra, R. (2015). Intimate Partner Violence Surveillance: Uniform Definitions and Recommended Data Elements. National Center for Injury Prevention and Control, Centers for Disease Control and Prevention, 1-164. https://doi.org/10.1136/bmj.328.7440.595

Burton, C. W., Halpern-Felsher, B., Rehm, R. S., Rankin, S. H., \& Humphreys, J. C. (2016). Depression and Self-Rated Health Among Rural Women Who Experienced Adolescent Dating Abuse: A Mixed Methods Study. Journal of Interpersonal Violence, 31(5), 920-941. https://doi.org/10.1177/0886260514556766

Caridade, S., Braga, T., \& Borrajo, E. (2019). Cyber dating abuse (CDA): Evidence from a systematic review. Aggression and Violent Behavior, 48(May), 152-168. https://doi.org/10.1016/j.avb.2019.08.018

Carlos, D. M., Campeiz, A. B., da Silva, J. L., Domingues Fernandes, M. I., da Cruz Leitão, M. N., Iossi Silv, M. A., \& Carvalho Ferriani, M. das G. (2017). School-based interventions for teen dating violence prevention: integrative literature review. Intervenciones En La Escuela Para Prevenir La Violencia En Las Relaciones Íntimas Entre Adolescentes: Revisión Integradora de La Literatura., 4(14), 133-145. Retrieved from 
http://10.0.49.163/RIV17030

Center for Disease Control and Prevention (CDC). (2019). Preventing Teen Dating Violence. Retrieved October 22, 2019, from https://www.cdc.gov/violenceprevention/intimatepartnerviolence/teendatingviolence/fastfac t.html

Das Gupta, M., Engelman, R., Levy, J., Luchsinger, G., Merrick, T., Rosen, J. E., ... Adviser, R. (2014). The State of World Population 2014 RESEARCHERS AND AUTHORS Other contributions from Sarah Castle UNFPA ADVISORY TEAM.

Draucker, C. B., Martsolf, D. S., Crane, S., McCord, A. L., Romero, L., \& Al-Khattab, H. A. (2019). A feasibility test of an online intervention to prevention dating violence in emerging adults. Archives of Psychiatric Nursing, 33(1), 37-45. https://doi.org/10.1016/j.apnu.2018.09.002

Duerksen, K. N., \& Woodin, E. M. (2019). Cyber Dating Abuse Victimization: Links With Psychosocial Functioning. Journal of Interpersonal Violence, 088626051987298. https://doi.org/10.1177/0886260519872982

Duval, A., Lanning, B. A., \& Patterson, M. S. (2018). A Systematic Review of Dating Violence Risk Factors Among Undergraduate College Students. Trauma, Violence \& Abuse, 1524838018782207-1524838018782207. https://doi.org/10.1177/1524838018782207

Foshee, V. A., Karriker-Jaffe, K. J., Reyes, H. L. M. N., Ennett, S. T., Suchindran, C., Bauman, K. E., \& Benefield, T. S. (2008). What Accounts for Demographic Differences in Trajectories of Adolescent Dating Violence? An Examination of Intrapersonal and Contextual Mediators. Journal of Adolescent Health, 42(6), 596-604. 
https://doi.org/10.1016/j.jadohealth.2007.11.005

Garthe, R. C., Sullivan, T. N., \& McDaniel, M. A. (2017). A meta-analytic review of peer risk factors and adolescent dating violence. Psychology of Violence, 45-57. Retrieved from http://10.0.4.13/vio0000040

Hébert, M., Daspe, M.-È., Lapierre, A., Godbout, N., Blais, M., Fernet, M., \& Lavoie, F. (2019). A Meta-Analysis of Risk and Protective Factors for Dating Violence Victimization: The Role of Family and Peer Interpersonal Context. Trauma, Violence \& Abuse, 20(4), 574590. Retrieved from http://10.0.4.153/1524838017725336

Hickman, L. J., Jaycox, L. H., \& Aronoff, J. (2004). Dating Violence among Adolescents: Prevalence, Gender Distribution, and Prevention Program Effectiveness. Trauma, Violence, \& Abuse, 5(2), 123-142. https://doi.org/10.1177/1524838003262332

Howard, D. E., Debnam, K. J., \& Wang, M. Q. (2013). Ten-year trends in physical dating violence victimization among U.S. adolescent females. The Journal of School Health, 83(6), 389-399. https://doi.org/10.1111/josh.12042

Howard, D. E., Debnam, K. J., Wang, M. Q., \& Gilchrist, B. (n.d.). 10-year trends in physical dating violence victimization among U.S. adolescent males. International Quarterly of Community Health Education, 32(4), 283-305. https://doi.org/10.2190/IQ.32.4.c

Johnson, R. M., LaValley, M., Schneider, K. E., Musci, R. J., Pettoruto, K., \& Rothman, E. F. (2017). Marijuana use and physical dating violence among adolescents and emerging adults: A systematic review and meta-analysis. Drug And Alcohol Dependence, 174, 47-57. https://doi.org/10.1016/j.drugalcdep.2017.01.012 
Johnson, R. M., Parker, E. M., Rinehart, J., Nail, J., \& Rothman, E. F. (2015). Neighborhood Factors and Dating Violence Among Youth: A Systematic Review. American Journal of Preventive Medicine, 49(3), 458-466. https://doi.org/10.1016/j.amepre.2015.05.020

Joly, L. E., \& Connolly, J. (2016). Dating violence among high-risk young women: A systematic review using quantitative and qualitative methods. Behavioral Sciences, 6(1). https://doi.org/10.3390/bs6010007

Jouriles, E. N., Garrido, E., Rosenfield, D., \& McDonald, R. (2009). Experiences of psychological and physical aggression in adolescent romantic relationships: Links to psychological distress. Child Abuse and Neglect, 33(7), 451-460. https://doi.org/10.1016/j.chiabu.2008.11.005

Liberati, A., Altman, D. G., Tetzlaff, J., Mulrow, C., Gotzsche, P. C., Ioannidis, J. P., ... Moher, D. (2009). The PRISMA statement for reporting systematic reviews and meta-analyses of studies that evaluate healthcare interventions: explanation and elaboration. Bmj, 339, b2700. https://doi.org/10.1136/bmj.b2700

Mcleroy, K. R., Bibeau, D., Steckler, A., \& Glanz, K. (1988). An Ecological Perspective on Health Promotion Programs. Health Education \& Behavior, 15(4), 351-377. https://doi.org/10.1177/109019818801500401

Munn, Z., Tufanaru, C., \& Aromataris, E. (2014). JBI's systematic reviews: data extraction and synthesis. The American Journal of Nursing, 114(7), 49-54. https://doi.org/10.1097/01.NAJ.0000451683.66447.89

O’Brien, K. M., Sauber, E. W., Kearney, M. S., Venaglia, R. B., \& Lemay, E. P. (2019). Evaluating the Effectiveness of an Online Intervention to Educate College Students About 
Dating Violence and Bystander Responses. Journal of Interpersonal Violence. https://doi.org/10.1177/0886260519829769

Park, S., \& Kim, S.-H. (2018). The power of family and community factors in predicting dating violence: A meta-analysis. Aggression \& Violent Behavior, 40, 19-28. https://doi.org/10.1016/j.avb.2018.03.002

Park, S., \& Kim, S.-H. (2019). Who Are the Victims and Who Are the Perpetrators in Dating Violence?: Sharing the Role of Victim and Perpetrator. Trauma, Violence \& Abuse, 20(5), 1524838017730648. https://doi.org/10.1177/1524838017730648

Park, Y., Mulford, C., \& Blachman-Demner, D. (2018). The Acute and Chronic Impact of Adolescent Dating Violence. In Adolescent Dating Violence (pp. 53-83). Elsevier. https://doi.org/10.1016/b978-0-12-811797-2.00003-7

Pedersen, P., \& Thomas, C. D. (1992). Prevalence and correlates of dating violence in a Canadian university sample. Canadian Journal of Behavioural Science / Revue Canadienne Des Sciences Du Comportement, 24(4), 490-501. https://doi.org/10.1037/h0078756

Rothman, E. F., McNaughton Reyes, L., Johnson, R. M., \& LaValley, M. (2012, January). Does the alcohol make them do it? Dating violence perpetration and drinking among youth. Epidemiologic Reviews. https://doi.org/10.1093/epirev/mxr027

Rubio-Garay, F., López-González, M. A., Carrasco, M. Á., \& Amor, P. J. (2017). The prevalence of dating violence: A systematic review. Papeles Del Psicólogo, 38(2), 135147. https://doi.org/10.23923/pap.psicol2017.2831

Spencer, C. M., Toews, M. L., Anders, K. M., \& Emanuels, S. K. (2019). Risk Markers for 
Physical Teen Dating Violence Perpetration: A Meta-Analysis. Trauma, Violence, \& Abuse, 152483801987570. https://doi.org/10.1177/1524838019875700

Stonard, K. E., Bowen, E., Lawrence, T. R., \& Price, S. A. (2014). The relevance of technology to the nature, prevalence and impact of Adolescent Dating Violence and Abuse: A research synthesis. Aggression and Violent Behavior, 19(4), 390-417. https://doi.org/10.1016/j.avb.2014.06.005

Taquette, S. R., \& Maia Monteiro, D. L. (2019). Causes and consequences of adolescent dating violence: a systematic review. Journal of Injury \& Violence Research, 11(2), 137-147. https://doi.org/10.5249/jivr.v11i2.1061

Teitelman, A. M., Ratcliffe, S. J., Dichter, M. E., \& Sullivan, C. M. (2008). Recent and past intimate partner abuse and HIV risk among young women. JOGNN - Journal of Obstetric, Gynecologic, and Neonatal Nursing, 37(2), 219-227. https://doi.org/10.1111/j.15526909.2008.00231.x

Temple, J. R., Choi, H. J., Elmquist, J. A., Hecht, M., Miller-Day, M., Stuart, G. L., ... WolfordClevenger, C. (2016). Psychological Abuse, Mental Health, and Acceptance of Dating Violence Among Adolescents. Journal of Adolescent Health, 59(2), 197-202. https://doi.org/10.1016/j.jadohealth.2016.03.034

The US Department of Justice. (n.d.). Dating Violence | OVW. Retrieved October 20, 2019, from https://www.justice.gov/ovw/dating-violence

Viejo, C., Monks, C. P., Sánchez, V., \& Ortega-Ruiz, R. (2016). Physical Dating Violence in Spain and the United Kingdom and the Importance of Relationship Quality. Journal of Interpersonal Violence, 31(8), 1453-1475. https://doi.org/10.1177/0886260514567963 
WHO. (2012). Understanding and addressing violence against women.

Wincentak, K., Connolly, J., \& Card, N. (2017). Teen dating violence: A meta-analytic review of prevalence rates. Psychology of Violence, 7(2), 224-241. https://doi.org/10.1037/a0040194

Zych, I., Viejo, C., Vila, E., \& Farrington, D. P. (2019). School Bullying and Dating Violence in Adolescents: A Systematic Review and Meta-Analysis. Trauma, Violence \& Abuse, 1524838019854460-1524838019854460. https://doi.org/10.1177/1524838019854460 
Appendix 1: Critical appraisal of the systematic reviews and meta-analyses

\begin{tabular}{|c|c|c|c|c|c|c|c|c|c|c|}
\hline $\begin{array}{l}\text { Author } \\
\text { and year } \\
\text { of } \\
\text { publicati } \\
\text { on }\end{array}$ & $\begin{array}{l}\text { Is the } \\
\text { review } \\
\text { question } \\
\text { clearly } \\
\text { and } \\
\text { explicitly } \\
\text { stated? }\end{array}$ & $\begin{array}{l}\text { Were the } \\
\text { inclusion } \\
\text { criteria } \\
\text { appropriate } \\
\text { for the review } \\
\text { question? }\end{array}$ & 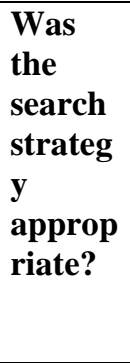 & $\begin{array}{l}\text { Were the } \\
\text { sources and } \\
\text { resources } \\
\text { used to } \\
\text { search for } \\
\text { studies } \\
\text { adequate? }\end{array}$ & $\begin{array}{l}\text { Were } \\
\text { the } \\
\text { criteria } \\
\text { for } \\
\text { appraisi } \\
\text { ng } \\
\text { studies } \\
\text { appropri } \\
\text { ate? }\end{array}$ & $\begin{array}{l}\text { Was critical } \\
\text { appraisal } \\
\text { conducted by } \\
\text { two or more } \\
\text { reviewers } \\
\text { independently } \\
?\end{array}$ & $\begin{array}{l}\text { Were the } \\
\text { methods } \\
\text { used to } \\
\text { combine } \\
\text { studies } \\
\text { appropriat } \\
\text { e? }\end{array}$ & $\begin{array}{l}\text { Was the } \\
\text { likelihood } \\
\text { of } \\
\text { publicatio } \\
\text { n bias } \\
\text { assessed? }\end{array}$ & $\begin{array}{l}\text { Were } \\
\text { recommendatio } \\
\text { ns for policy } \\
\text { and/or practice } \\
\text { supported by } \\
\text { the reported } \\
\text { data? }\end{array}$ & $\begin{array}{l}\text { Were the } \\
\text { specific } \\
\text { directives } \\
\text { for new } \\
\text { research } \\
\text { appropriat } \\
\text { e? }\end{array}$ \\
\hline $\begin{array}{l}\text { (Rothma } \\
\text { n et al., } \\
\text { 2012) }\end{array}$ & Yes & Yes & Yes & Yes & Not sure & Yes & Yes & Yes & N/A & Yes \\
\hline $\begin{array}{l}\text { (Stonard } \\
\text { et al., } \\
\text { 2014) }\end{array}$ & Yes & Yes & Yes & Yes & Yes & No & Yes & N/A & Yes & Yes \\
\hline $\begin{array}{l}\text { (Johnso } \\
\text { n et al., } \\
2015)\end{array}$ & Yes & Yes & Yes & Not sure & Yes & No & Yes & N/A & Yes & Yes \\
\hline $\begin{array}{l}\text { (Joly \& } \\
\text { Connoll } \\
\text { y, 2016) }\end{array}$ & Yes & Not sure & Yes & Yes & Not sure & No & Yes & No & N/A & Yes \\
\hline $\begin{array}{l}\text { (Rubio- } \\
\text { Garay et } \\
\text { al., } \\
\text { 2017) }\end{array}$ & Yes & Not sure & Yes & Yes & Yes & No & Yes & No & Yes & No \\
\hline $\begin{array}{l}\text { (Garthe } \\
\text { et al., } \\
2017)\end{array}$ & Yes & Yes & Yes & Yes & Yes & No & Yes & N/A & Yes & Yes \\
\hline $\begin{array}{l}\text { (Johnso } \\
\text { n et al., } \\
2017)\end{array}$ & Yes & Yes & Yes & Not sure & Yes & No & Yes & Yes & Yes & Yes \\
\hline $\begin{array}{l}\text { (Wincen } \\
\text { tak et } \\
\text { al., } \\
\text { 2017) }\end{array}$ & Yes & Yes & Yes & Yes & Yes & Yes & Yes & Yes & Yes & Yes \\
\hline $\begin{array}{l}\text { (Duval } \\
\text { et al., } \\
2018 \text { ) }\end{array}$ & Yes & Yes & Yes & Yes & Yes & Yes & Yes & N/A & Yes & Yes \\
\hline
\end{tabular}




\begin{tabular}{|c|c|c|c|c|c|c|c|c|c|c|}
\hline $\begin{array}{l}\text { (S. Park } \\
\text { \& Kim, } \\
\text { 2018) }\end{array}$ & Yes & Yes & Yes & Yes & Yes & Yes & Yes & Yes & Yes & $\mathrm{N} / \mathrm{A}$ \\
\hline $\begin{array}{l}\text { (Carida } \\
\text { de et al., } \\
2019)\end{array}$ & Yes & Yes & Yes & Yes & Yes & No & N/A & N/A & N/A & Yes \\
\hline $\begin{array}{l}\text { (Hébert } \\
\text { et al., } \\
2019)\end{array}$ & Yes & Yes & Yes & Yes & Yes & No & Yes & Yes & Yes & Yes \\
\hline $\begin{array}{l}\text { (S. Park } \\
\text { \& Kim, } \\
\text { 2019) }\end{array}$ & Yes & Yes & Yes & Yes & Yes & Yes & Yes & Yes & Yes & Yes \\
\hline $\begin{array}{l}\text { (Spencer } \\
\text { et al., } \\
\text { 2019) }\end{array}$ & Yes & Yes & Yes & Yes & Not Sure & No & Yes & Yes & Yes & Yes \\
\hline $\begin{array}{l}\text { (Taquett } \\
\text { e \& } \\
\text { Maia } \\
\text { Monteir } \\
\text { o, 2019) }\end{array}$ & Yes & Yes & $\begin{array}{l}\text { Not } \\
\text { sure }\end{array}$ & No & N/A & Yes & Not sure & N/A & Yes & Yes \\
\hline $\begin{array}{l}\text { (Zych et } \\
\text { al., } \\
\text { 2019) }\end{array}$ & Yes & Yes & Yes & Yes & N/A & Yes & Yes & Yes & Yes & Yes \\
\hline
\end{tabular}

